\title{
11.02
}

\section{Plano de Gerenciamento dos Resíduos Sólidos de Serviços de Saúde de uma unidade de Saúde da Família ${ }^{1}$}

\author{
Solid Health Services Waste Management Plan in a Family Health unity.
}

\section{Joceli Custodia de Assunção}

Tecnólogo em Gestão Ambiental. Universidade Norte do Paraná (UNOPAR). Cáceres-MT, Brasil.

\section{Solange da Silva Lima}

Especialista em Gestão em Saúde. Docente auxiliar da Universidade do Estado de Mato Grosso (UNEMAT) campus de Cáceres. Cáceres-MT, Brasil.

\section{Marilda Soares da Silva}

Bacharel em Enfermagem, Especialista em Saúde da Família. Secretaria de Saúde de Tangará da Serra-MT, Brasil.

\begin{abstract}
Resumo: Objetivo: $O$ presente trabalho objetivou identificar as medidas realizadas na unidade de saúde da família do município de Tangará da Serra-MT, para o gerenciamento dos resíduos sólidos. Metodologia: A pesquisa foi realizada em uma das unidades de saúde da família pertencente a cidade de Tangará da Serra-MT e foi escolhida usando o método aleatório com o período de análise que transcorreu de janeiro a outubro de 2012. Para a coleta de dados utilizou-se de um cheklist elaborado pela autora com base na fundamentação bibliográfica sobre a existência do Plano de Gerenciamento de Resíduos Sólidos dos Serviços de Saúde (PGRSSS). Resultados: A unidade de saúde da família não apresentava a coleta externa conforme a determinação da RDC 306/04, bem como a inexistência de educação continuada sobre o tema. Conclusões: Os dados obtidos apontam para a necessidade de um maior e mais efetivo envolvimento de gestores e profissionais de saúde com essa questão, visando não somente proporcionar maior segurança para os trabalhadores no exercício de suas funções, mas também contribuir para a preservação do meio ambiente e para a saúde da população. Vale ressaltar que os gestores municipais devem estar atentos em atender o que preconiza a legislação sobre o Plano de Gerenciamento de Resíduos Sólidos dos Serviços de Saúde PGRSSS conforme preconiza a RDC vigente bem como a necessidade de pesquisas mais específicas sobre o tema entre os funcionários da unidade e maior fiscalização que irá reforçar sobremaneira a importância da não contaminação e da contaminação do solo que irá favorecer a preservação ambiental.
\end{abstract}

Palavras chave: Gerenciamento de resíduos; resíduos de serviços de saúde; Unidades de Saúde da Família.

1 Recorte do Trabalho de Conclusão de Curso apresentado à Universidade Norte do Paraná (UNOPAR) para a obtenção do título de Tecnólogo em Gestão Ambiental. 
Key-words: Waste management; health services waste; family health units.

\section{Introdução}

Segundo pesquisa do Instituto Brasileiro de Geografia e Estatística (IBGE) 45\% dos municípios brasileiros não possuem coleta especial para resíduos de serviços de saúde, tampouco separação do lixo doméstico, confrontando com 6,4\% dos que recebem tratamento e destino adequado. (IBGE, 2005).

O impacto do lixo produzido pelo descarte inadequado dos resíduos dos serviços de saúde em especial corroboram para a falta de saneamento básico presente em muitos municípios, e que conforme IBGE são responsáveis por $80 \%$ das doenças e 65 \% das internações hospitalares.

Conforme regulamentação publicada pela Agência Nacional de Vigilância Sanitário (ANVISA), por meio da RDC 306/04 entende-se por geradores de resíduos sólidos de saúde:

[...] todos os serviços relacionados com o atendimento à saúde humana ou animal, inclusive os serviços de assistência domiciliar e de trabalhos de campo; laboratórios analíticos de produtos para saúde; necrotérios, funerárias e serviços onde se realizem atividades de embalsamamento (tanatopraxia e somato conservação); serviços de medicina legal; drogarias e farmácias inclusive as de manipulação; estabelecimentos de ensino e pesquisa na área de saúde; centros de controle de zoonoses; distribuidores de produtos farmacêuticos, importadores, distribuidores e produtores de materiais e controles para diagnóstico in vitro; unidades móveis de atendimento à saúde; serviços de acupuntura; serviços de tatuagem, dentre outros similares (RDC, 2004, pág. 13).

O Plano de Gerenciamento de Resíduos de Serviços de Saúde tem por objetivo principal contribuir para a melhor segregação dos resíduos promovendo a redução do seu volume e diminuir a incidência de acidentes ocupacionais através a educação continuada (UNIMED, 2007).

Compreende o gerenciamento dos RRS o conjunto de ações voltadas ao planejamento através de bases científicas respalda em normativas e leis, com objetivo de proporcionar segurança, eficiência e proteção aos trabalhadores durante o encaminhamento dos mesmos, contribuindo para a saúde pública e preservação dos recursos naturais e do meio ambiente. (RDC Anvisa n³06/04). 
Dentre os diversos grupos que compõem os resíduos sólidos contaminantes, merecem atenção os imunobiológicos por se tratarem de materiais descartados já que os mesmos apresentam importante risco de infecção. Conforme Ministério da Saúde os imunobiológicos são compostos por microorganismos vivos atenuados (vacinas contra: sarampo, poliomielite, febre amarela, tuberculose etc.) e por bactérias e vírus mortos ou obtidos por engenharia genética (vacinas como DTP, dT, DT, Hib, HB etc.), sendo os primeiros descartados após tratamento prévio especial (de redução de carga microbiana) antes do descarte final.

Nesse contexto vale salientar a importante do tema relativo aos imunobiológicos e os resíduos sólidos de serviços de saúde, uma vez que a informação a respeito do descarte correto ainda é motivo de indagações por parte dos profissionais da área.

\section{Objetivo}

\section{a. Objetivo geral}

- Descrever e caracterizar as diversas formas de descartes dos resíduos de serviços de saúde provenientes de imunização em uma Unidade de Saúde da Família.

\section{b. Objetivos específicos}

- Identificar a existência do Plano de Gerenciamento dos Serviços de Saúde (PGRSS)

- Conhecer as atividades realizadas para a segregação, acondicionamento e transporte dos resíduos gerados na unidade em questão

- Analisar as facilidades e dificuldades encontradas pelos trabalhadores de saúde da Unidade pesquisada no gerenciamento dos resíduos sólidos dos serviços de saúde.

\section{$3 \quad$ Metodologia}

\section{a. Área de estudo}

Trata-se de um estudo elaborado a partir de pesquisa de levantamento, do tipo descritivo, exploratória, e de análise qualitativa. Utilizada para descrever e sintetizar 
os dados relativos ao gerenciamento e descarte dos imunobiológicos de uma unidade de Saúde da Família.

\section{b. Coleta de dados}

A referida pesquisa realizou-se na Unidade de Saúde da Família Tangará II, no município de Tangará da Serra, localizada nos limites do Bairro Jardim Califórnia e Jardim Tangará II.

Escolheu-se a Unidade de Saúde da Família Tangará II pela facilidade de acesso para a pesquisadora, tendo em vista a possibilidade de coletar dados em outras unidades.

\section{Instrumento de Coleta de Dados}

Instrumento de observação direta com check-list contendo normas regulamentadas pela Anvisa, Conama, Ministério da Saúde e ABNT, contando com 20 itens.

\section{d. Análise dos Dados}

Os dados foram tabulados na forma de tabelas e gráficos e assim apreciados com uma análise respaldada em literaturas diversas referentes ao tema abordado no estudo para o trabalho de término de conclusão de curso, e acrescida das reflexões da autora.

\section{Resultados e discussão}

O instrumento de coletas de dados foi pautado nas diferentes fases do processo de descarte dos resíduos sólidos. Nesse instrumento, foram contempladas as principais etapas do processo de descarte: armazenamento interno e segregação na unidade de saúde, preparo dos imunobiológicos, coleta externa e existência de educação continuada.

O armazenamento interno e a segregação dos resíduos provenientes das salas de vacinação devem acontecer respeitando as características dos produtos e validade dos mesmos, conforme as normativas da RDC 306.

Pela presente pesquisa foi constatada que a unidade de saúde escolhida como local do estudo atendeu a $100 \%$ dos critérios relacionados ao armazenamento interno e segregação dos resíduos. Dessa forma, verifica-se que existe por parte da equipe de saúde esforço efetivo no controle de saúde e adequação às normas vigentes. 
Na etapa referente ao preparo dos imunobiológicos, é importante destacar que mesmo tendo sido utilizado apenas um item para avaliação, a unidade de saúde obteve resultado de $100 \%$ de adequação. Tendo em vista, que neste setor há apenas a indicação de preparo apenas para vacinas de vírus vivos e atenuados, não se faz possível avaliar outros instrumentos de preparo.

Na etapa referente à coleta externa, foram relacionados 6 itens utilizados como critério de avaliação. Esta etapa compreende a remoção dos RSS do abrigo de resíduos (armazenamento externo) até a unidade de tratamento ou disposição final, utilizando-se técnicas que garantam a preservação das condições de acondicionamento e a integridade dos trabalhadores, da população e do meio ambiente, devendo estar de acordo com as orientações dos órgãos de limpeza urbana. (RDC 306/2004).

Neste item, os resultados referentes à coleta externa foram de $66,6 \%$ de atendimento aos itens utilizados na avaliação, não sendo obedecida a utilização de óculos de proteção visual dos profissionais responsáveis pela coleta externa e a existência de aterro sanitário adequado no município.

A utilização de óculos de proteção visual serve como medida preventiva de acidentes com gotículas de resíduos de natureza química e biológica. $\mathrm{O}$ trabalho ainda constatou que há a aplicação do preparo adequado aos materiais classificados como pertencente ao grupo $A 1$, verificado pela existência do tratamento de autoclavagem dos imunobiológicos de vírus vivos e atenuados, conforme determinação do Ministério da Saúde (2001).

A coleta externa que também faz parte do processo de manejo dos resíduos também foi avaliada no presente trabalho, tendo obtido o resultado de $25 \%$ de atendimento das normativas utilizadas no instrumento de coleta. Desta forma, fica evidente que não o cumprimento adequado no que se refere à coleta externa.

Conforme Takada (2003) os problemas relacionados aos resíduos sólidos de saúde envolvem dois principais problemas: um - o funcionário das unidades e o outro a visão sócio-ambiental e sanitária, associada ao destino final destes materiais. Com isso é evidente que a importância do tratamento adequado aos resíduos sólidos é de extrema importância para a saúde pública da população diretamente envolvida no problema. 
Por fim, num plano de gerenciamento de resíduos sólidos, é necessária a capacitação contínua de todos os envolvidos no processo de geração e descarte dos resíduos sólidos. (RDC 306/2004).

O estudo verificou que o município não implementou nenhuma ação de educação continuada voltada ao Plano de Gerenciamento de Resíduos Sólidos, não atendendo a este quesito conforme é preconizado pela Regulamentação da Anvisa por meio da RDC 306/04.

\section{Considerações finais}

Tendo em vista as informações apresentadas e discutidas no presente estudo, pode-se traçar um panorama da situação da Unidade de Saúde da Família pesquisada no que se tange ao gerenciamento dos resíduos sólidos produzidos pelas mesmas, a partir do qual se constata que a unidade, em sua totalidade, apresenta falhas, em relação ao manejo adequado dos resíduos, aos quais se deve em grande parte, à falta de treinamento dos recursos humanos e a escassez de recursos materiais adequados.

Destaca-se, sobremaneira, a ausência de um plano formal para gerenciamento dos resíduos produzidos nas unidades, bem como a falta de um treinamento específico para o desenvolvimento dessa atividade.

Os dados obtidos apontam para a necessidade de um maior e mais efetivo envolvimento de gestores e profissionais de saúde com essa questão, visando não somente proporcionar maior segurança para os trabalhadores no exercício de suas funções, mas também contribuir para a preservação do meio ambiente e para a saúde da população.

Pro fim, é imprescindível lembrar a importância do tema, e a necessidade de estudos permanentes e específicos, já que o tema Planejamento e Gerenciamento de Resíduos Sólidos dos Serviços de Saúde é de natureza abrangente, carecendo de apreciações através de estudos científicos contínuos.

\section{Referências}

Plano de Gerenciamento dos Resíduos de Serviços de Saúde da Sociedade Beneficente São Vicente de São Paulo. Manual técnico disponível no endereço: http://dx.doi.org/10.1590/S1413-41522011000200005. 
AGÊNCIA NACIONAL DE VIGILÂNCIA SANITÁRIA. RDC n³0 6, de 07 de dezembro de 2004. Dispõe sobre o Regulamento Técnico para gerenciamento de resíduos de serviços de saúde disponível no endereço: http://www4.anvisa.gov.br/base/visadoc/CP/CP\%5B20735-1-0\%5D.PDF;

CONAMA, RDC $n^{\circ} 358$, de 29 de abril de 2005. Dispõe sobre o tratamento e a disposição final dos resíduos de serviços de saúde e dá providências. Disponível no endereço: http://www.mma.gov.br/port/conama/res/res05/res35805.pdf;

IBGE-www.ibge.gov.br

MINISTÉRIO DA SAÚDE. Manual de Normas de Vacinação. 3. ed. Brasília : Fundação Nacional de Saúde, 2001. 72p.

SILVA, Carlos Augusto Machado da Costa et al. Caracterização microbiológica de lixiviados gerados por resíduos sólidos domiciliares e de serviços de saúde da cidade do Rio de Janeiro. Engenharia Sanitária e Ambiental, 16(2):127-132, 2011. [on line]. Disponível em: http://dx.doi.org/10.1590/S1413-41522011000200005.

SALES, Carla Cristina de Lima; SPOLTI, Gracieli Pâmela; LOPES, Maria do Socorro Bezerra e LOPES, David Franco. Gerenciamento dos resíduos sólidos dos serviços de saúde: aspectos do manejo interno no município de Marituba, Pará, Brasil. Ciência \& Saúde Coletiva, 14(6):2231-2238, 2009. [on line]. Disponível em: http://dx.doi.org/10.1590/S1413-81232009000600032.

SIQUEIRA, Mônica Maria e MORAES, Maria Silvia de. Saúde coletiva, Resíduos Sólidos Urbanos e os Catadores de Lixo. Ciência \& Saúde Coletiva, 14(6):2115-2122, 2009. [on line]. Disponível em: http://dx.doi.org/10.1590/S1413-81232009000600018.

TAKADA, A.C.S. O Plano de Gerenciamento de Resíduos de Serviços de Saúde e o Direito do Trabalhador. Monografia. Escola de Saúde Pública. $1^{\underline{a}}$ ed. Fundação Osvaldo Cruz. Brasília : Fiocruz, 2003. 